\title{
Expression of a Testis-Specific Nuclear Protein, TRA98, in Mouse Testis during Spermatogenesis. A Quantitative and Qualitative Immunoelectron Microscopy (IEM) Analysis
}

\author{
Natsuki Inoue, Yuko Onohara, Sadaki Yokota* \\ Section of Functional Morphology, Faculty of Pharmaceutical Sciences, Nagasaki \\ International University, Nagasaki, Japan \\ E-mail: ${ }^{*}$ syokota@niu.ac.jp \\ Received November 2, 2011; revised December 8, 2011; accepted December 13, 2011
}

\begin{abstract}
TRA98 is a testis-specific nuclear protein, but its biological role is unclear. We analyzed the localization of TRA98 in developing spermatogenic cells using immunofluorescence (IF) and immunoelectron microscopy (IEM). TRA98 was localized exclusively to the nuclei. In spermatocytes, IF staining was associated with certain sub-nuclear structures to show a reticular pattern; the XY body was strongly stained. In spermatids, both reticular and punctate staining patterns were observed. In late spermatids, staining decreased as cell differentiation proceeded. However, epididymal sperm were strongly stained when smear preparations were not fixed, or followed by treatment with $4 \mathrm{M}$ urea or $\%$ mercaptoethanol. IEM showed that gold signals were closely associated with electron-dense masses but not with the nucleoli. We then investigated the expression of TRA98 in differentiating spermatocytes using a quantitative IEM technique. A small expression peak was observed around stage II-III and a second large peak was noted at stage XI. In spermatids, a single expression peak was observed at step 5; labeling density then decreased gradually but did not reach zero. In early spermatids, heterochromatin was stained much more than euchromatin. The results suggest that the function of TRA98 increases at three points during spermatogenesis. In addition, TRA98 is maintained in the sperm head and carried into the egg after fertilization.
\end{abstract}

Keywords: Nuclear Protein, TRA98, Testis, Stage Cycle, Immunocytochemistry

\section{Introduction}

Spermatogenesis is a dynamic and highly complicated process that may be divided into three phases based on functional aspects: 1) the proliferative phase in which spermatogonia undergo rapid successive cell divisions; 2) the meiotic phase in which recombination and segregation of chromosomes occur, 3) the differentiation phase in which spermatids transform into spermatozoa [1-3]. During these phases, numerous stage-specific genes as well as house-keeping ones are expressed and supply the proteins required for stage-specific functions and the usual metabolism of testis [4]. The differentiation of spermatogenic cells appears to be regulated by the elaborate system with which many nucleus-resident proteins are thought to be concerned.

TRA98 was identified as a nucleus-resident protein in mouse testis germ cells and the antibody to it has been prepared by Tanaka et al. [5]. The biological function of TRA98 is unknown. In the present paper, we study two issues: 1) the localization of TRA98 in the nuclei of spermatogenic cells of adult mice using immunofluorescence (IF) and immunoelectron microscopy (IEM); and 2) the expression of TRA98 in spermatocytes and spermatids during spermatogenesis, as revealed by a quantitative (IEM).

\section{Materials and Methods}

\subsection{Animals}

Mice (SD, 20 - 25 g) were obtained from Kyudo (Tosu, Japan) and fed appropriate standard diets and water ad libitum until use. The animal experiments were performed in accordance with the guidance for animal experiments issued by Nagasaki International University. 


\subsection{Antibodies and Related Probes}

Rat monoclonal anti-TRA98 antibody was a generous gift from Dr. Tanaka (Faculty of Pharmaceutical Sciences, Nagasaki International University). Protein Agold probe (15 nm gold) was prepared following the method of de Roe et al. [6]. Rabbit anti-rat IgG was purchased from DAKO (Tokyo, Japan). Goat anti-rat IgG (heavy and light chains) labeled with Ultra Small Immunogold reagent was obtained from AURION (Wageningen, the Netherlands). Rabbit anti-mitochondrial isocitrate dehydrogenase (ICD1) was as described previously [7]. Rabbit anti-histone $\mathrm{H} 2 \mathrm{~B}$, rabbit anti-protein disulfide isomerase (PDI), and rabbit anti- $\alpha$-tubulin were obtained from Cell Signaling Technology (Boston, MA, USA), Sigma-Aldrich (Tokyo, Japan), and Thermo Fisher Scientific (Fremont, CA, USA), respectively.

\subsection{Western Blotting}

Mouse testes were homogenized in $5 \mathrm{mM}$ MOPS-KOH buffer (pH 7.4) containing $0.25 \mathrm{M}$ sucrose, $1 \mathrm{mM}$ EDTA, $1 \mathrm{mM}$ phenylmethylsulfonyl fluoride (PMSF) and a cocktail of protease inhibitors $(1 \mu \mathrm{g} / \mathrm{ml})$, including leupeptin, pepstatin, aprotinin and antipain (medium A). The homogenate was filtered through a nylon filter with mesh size $100 \mu \mathrm{m}$ and the filtrate was centrifuged at 800 $g$ for $2 \mathrm{~min}$. The precipitate was suspended in medium A and used for isolation of nuclei as mentioned below. The supernatant fraction was centrifuged at $10,000 \mathrm{~g}$ for 20 min and the pellet (mitochondrial fraction) was suspended in a small volume of medium A. The supernatant fraction was centrifuged at $100,000 \mathrm{~g}$ for $60 \mathrm{~min}$ with a Beckman ultracentrifuge using a SW 41 swing rotor. The resulting pellets were suspended in medium $\mathrm{A}$ and used as the microsomal fraction, while the supernatant fraction was used as the cytosol fraction. The cell fractions were stored at $-70^{\circ} \mathrm{C}$. Protein concentrations were determined by the bicinchoninic acid method (Pierce Chemical, Rockford, IL, USA) using bovine serum albumin (BSA) as a standard. The protein concentrations of the fractions were adjusted to $1 \mathrm{mg} / \mathrm{ml}$, mixed with one volume of sample buffer for SDS-PAGE, and heated in boiling water for $5 \mathrm{~min}$. Cell fraction samples were analyzed by western blotting. The molecular mass of TRA98 was estimated with reference to prestained protein makers (Nippon Genetics Europe, Dueren, Germany).

\subsection{Dot Blot Analysis of TRA98 Content in Cell Fractions of Mouse Testis}

The cell fractions including mitochondria, microsomes, and cytosol were isolated from mouse testes homogenate by differential centrifugation as described above. Nuclei were isolated by the method of Rickwood and Ford [8]. Briefly, the crude nuclear fraction (800 g pellet) was suspended in medium A containing 1\% Triton X-100 and centrifuged at $800 \mathrm{~g}$ for $10 \mathrm{~min}$. The resulting pellet was suspended in $2 \mathrm{ml}$ of medium $\mathrm{A}$, mixed with $72.5 \%$ (wt/vol) metrizamide (Sigma-Aldrich) in medium A without $0.25 \mathrm{M}$ sucrose and centrifuged at $10,000 \mathrm{~g}$ for 20 $\min$ at $5^{\circ} \mathrm{C}$. The nuclei-rich pellicles at the surface of the metrizamide solution were collected, suspended in a $\mathrm{x} 10$ volume of medium $\mathrm{A}$, and then centrifuged at $6000 \mathrm{~g}$ for $10 \mathrm{~min}$. The pellet was suspended in $1.6 \mathrm{ml}$ of medium A. Each fraction was diluted by medium A. The diluted fractions $(90 \mu \mathrm{l})$ were mixed with $90 \mu \mathrm{l}$ SDS-PAGE sample buffer and $20 \mu \mathrm{l} 0.3 \mathrm{M}$ iodoacetamide, treated in boiling water for $5 \mathrm{~min}$, and centrifuged at $10,000 \mathrm{~g}$ for $10 \mathrm{~min}$. The supernatant fractions were diluted 1000 -fold and 1 - $5 \mu \mathrm{l}$ was loaded onto PVDF membranes for immunoblotting. TRA98 was visualized using combination of HRP-labeled rabbit anti-rat IgG and 3,3'-diaminobenzidine (DAB) reaction. Internal standard proteins, histone 2A (H2A, nuclei), mitochondrial isocitrate dehydrogenase (ICD1, mitochondria), protein disulfide isomerase (PDI, microsomes), and $\alpha$-tubulin (cytosol) were visualized using a combination of rabbit antibodies against each protein, HRP-labeled goat anti-rabbit $\mathrm{IgG}$, and $\mathrm{DAB}$ reaction. The staining intensity was measured using a densitometer. The total amount of DDX25 in each cell fraction was calculated as follows: the densitometric values obtained were multiplied by the final volume of each fraction. The data were obtained from three measurements and the average values and standard deviations were plotted.

\subsection{IF Staining}

\subsubsection{Staining of Testis Tissue Sections}

Mouse testes were embedded in Tissue-Tek (SAKURA Finetec, Tokyo, Japan) and frozen in isopentane cooled by liquid nitrogen. Frozen sections ( $6 \mu \mathrm{m}$ thickness) were cut with a Leitz cryostat (Leica Instruments, Nussloch, Germany) and picked up on silicone-coated glass slides. Before drying, sections were fixed in a fixative consisting of $4 \%$ paraformaldehyde (PF), $0.01 \% \mathrm{CaCl} 2$, and $0.1 \mathrm{M}$ HEPES-KOH buffer $(\mathrm{pH} \mathrm{7.4)}$ for $15 \mathrm{~min}$ at room temperature (RT). Sections were treated with $0.2 \%$ Triton X-100-phosphate-buffered saline (PBS) for $15 \mathrm{~min}$ and then incubated in blocking solution containing $2 \%$ fish gelatin and $15 \mathrm{mM} \mathrm{NaN3}$ in PBS for $30 \mathrm{~min}$. Sections were then incubated with anti-TRA98 antibody (x 1000 ) overnight at $4^{\circ} \mathrm{C}$. After washing in PBS, sections were incubated with Alexa 488- or 568-labeled goat anti-rat IgG solutions containing $3 \mu \mathrm{M}$ DAPI (Hoechst, 
Tokyo, Japan) for $60 \mathrm{~min}$ at RT. For the IF control, unrelated rat serum was used for the primary reaction, followed by Alexa 488- or 568-labeled secondary antibodies. The sections were examined with a Nikon Eclipse E600 fluorescence microscope (Nikon, Tokyo, Japan). The images were merged using Adobe Photoshop7.0 to visualize cell contours. Seminiferous tubules stage was judged from the size and shape of spermatocytes and spermatids stained by DAPI with reference to the stages of the cycle illustrated by Russell and coworkers [9].

\subsubsection{Staining of Smear Preparation of Epididymal Sperm}

Mouse sperm were collected from the caudal epididymis and suspended in Hank's solution. The sperm were smeared on silicon-coated glass slides, dried in air and stored at $-20^{\circ} \mathrm{C}$ until use. The smear preparations were treated with various solutions, including $2 \mathrm{M}$ guanidine- $\mathrm{HCl}$ in $0.25 \mathrm{M}$ Tris- $\mathrm{HCl}$ buffer ( $\mathrm{pH} 8.5$ ), $4 \mathrm{M}$ urea in PBS, 2\% mercaptoethanol in PBS, $2 \mathrm{M}$ guanidine- $\mathrm{HCl}$ $+2 \%$ mercaptoethanol, $4 \mathrm{M}$ urea $+2 \%$ mercaptoethanol, for $30 \mathrm{~min}$ at RT. After washing in PBS, the preparations were treated with $2 \%$ fish gelatin in PBS for $30 \mathrm{~min}$, and then incubated with rat anti-TRA98 antibody (x 1000) overnight at $4{ }^{\circ} \mathrm{C}$. The primary antibody was visualized by Alexa 568-labeled goat anti-rat IgG. DNA was stained by DAPI. The preparations were observed as described above.

\subsection{IEM}

Testes of mice were cut into small tissue blocks in icecold fixatives and fixed at $4^{\circ} \mathrm{C}$ for $1 \mathrm{~h}$. The fixatives used were 1) $4 \% \mathrm{PF}$ in $0.05 \mathrm{M}$ HEPES-KOH (pH 7.4); 2) $4 \%$ $\mathrm{PF}+0.2 \%$ glutaraldehyde $(\mathrm{GA})+0.01 \% \mathrm{CaCl}_{2}$ in the same buffer; and 3) $3 \% \mathrm{PF}+0.01 \% \mathrm{GA}+0.01 \% \mathrm{CaCl}_{2}$. Tissue blocks were washed in PBS and dehydrated with a graded series of ethanol concentrations and embedded in LR White (London Resin, Reading, UK) at $-20^{\circ} \mathrm{C}$. The resin was polymerized by UV irradiation at $-20^{\circ} \mathrm{C}$. Thin sections of LR White-embedded testis tissues were cut with a diamond knife equipped with a Reichert Ultracut R, mounted on nickel grids, and incubated with rat anti-TRA98 overnight at $4^{\circ} \mathrm{C}$. For immunocytochemical control, unrelated rat serum was used instead of the primary specific antibody. Sections were then incubated with goat anti-rat IgG labeled with Ultra Small colloidal gold (AURION, Wageningen, the Nether- lands), followed by silver enhancement using AURION R-Gent SE-EM. Some sections were incubated with rabbit antirat IgG, followed by protein A-gold (15 nm gold). Sections were contrasted and then examined with a Hitachi H7650 electron microscope (Hitachi, Tokyo, Japan) at an acceleration voltage of $80 \mathrm{kV}$. Stage of seminiferous tubules and step of spermatids were judged according to stages of the cycle illustrated by Russell and coworkers [9].

\subsection{Quantification of Silver Labeling}

Ten IEM digital pictures of each seminiferous epithelium at stages I to XII were taken at magnification of x 2000 and printed on A4 paper. The areas of the nucleus and cytoplasm were measured by the method of Weibel and coworkers [10] and the silver grains in each area were counted. The labeling density in each area was calculated as silver grains per $\mu \mathrm{m}^{2}$.

\subsection{Statistical Analyses}

The mean and SEM were calculated for all study parameters. Statistical analyses were carried out by use of student's $t$-test for unpaired observations. $P$ values $<0.05$ were considered statistically significant.

\section{Results}

\subsection{Western Blotting and Distribution of TR98 in Cell Fractions}

A band corresponding to a molecular mass of approximately $100 \mathrm{kDa}$ was observed in mouse testis homogenate and in the nuclear fraction, but not in the other cell fractions (Figure 1(A)). Dot blotting analysis showed that most of the TRA98 was contained in the nuclear fraction (Figure 1(B)). A very small amount of TRA98 was detected in the microsomes (approximately 3\%). Marker proteins, H2A), ICD1, PDI, and $\alpha$-tubulin were detected prominently in the nuclear fraction, mitochondria, microsomes, and cytosol, respectively (Figure 1(B)).

\subsection{IF Staining}

The nuclei of spermatogenic cells, including spermatogonia, spermatocytes and round spermatids, were stained for TRA98 with various staining intensities (Figure 2). Spermatids at the later step 12 were almost negative after treatment of frozen sections with $0.2 \%$ Triton $\mathrm{X} 100$. Strong staining was noted as one or three small spots in the nuclei of spermatids at step VI-VII (Figure 3(A)). A reticular staining pattern was noted in the nucleoplasm of mid and late pachytene spermatocytes and a region corresponding to a sex body was strongly stained in some of the cells (Figures 2(B) and (C)). These positive-staining reactions were not observed in the IF con- 


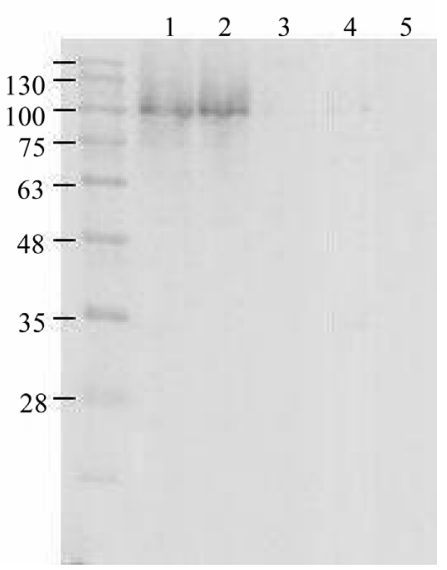

(A)

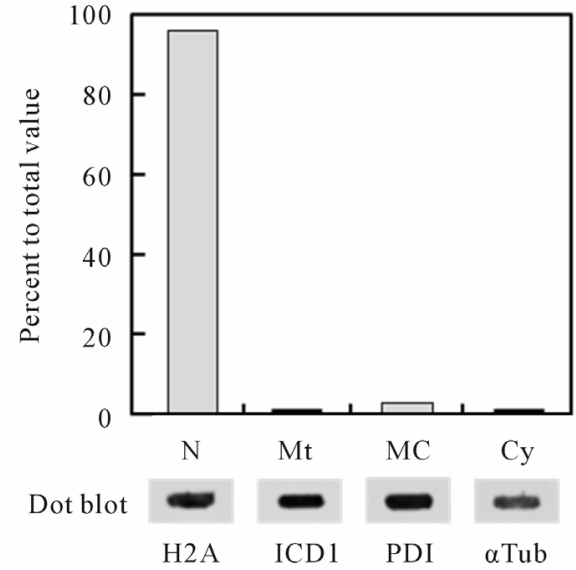

(B)

Figure 1. (A) Western blotting analysis of mouse testis cell fractions: lane 1, homogenate; lane 2, nuclei; lane 3, mitochondria; lane 4, microsomes; lane 5, cytosol. (B) Dot blotting distribution of TRA98 in the cell fractions of mouse testis. N, nuclei; Mt, mitochondria; $\mathrm{Cy}$, cytosol; MC, microsomes. Dot blot: signals of internal standard protein for each cell fraction. H2A, histone H2A for nucleus; ICD1, isocitrate dehydrogenase for mitochondria; PDI, protein disulfide isomerase for ER; $\alpha$-Tub, $\alpha$-tubulin for cytosol.
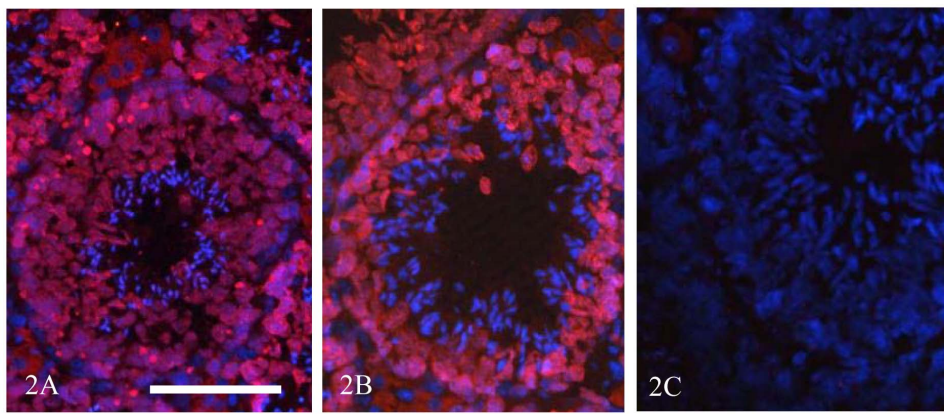

Figure 2. IF staining of mouse testis for TRA98. A. Seminiferous tubule at stage I. B. Seminiferous tubule at stage VIII. Note that TRA98 staining is observed in the nuclei of spermatogenic cells. Magnification A-C: $\times 360$. Bar $=50 \mu \mathrm{m}$.

trol sections incubated with IgG fraction from non- immunized rat, followed by Alexa 488 or 568 -labeled goat anti-rat $\operatorname{IgG}$ (Figure 2(C)).

The nuclei of spermatids at later steps of 12 were not stained after treatment with $0.2 \%$ Triton X-100 (Figures 2 and 3). We tried to treat smear preparation of epididymal sperms with various media as described in Materials and methods. After treatment of unfixed smear preparations with $4 \mathrm{M}$ urea, $2 \mathrm{M}$ guanidine hydrochloride or $2 \%$ mercaptoethanol, sperm heads were stained for TRA98, but sperm heads fixed with 4\% PF were not stained after any treatment (Figure 4). Thus, TRA98 in condensed nuclei was shown to be very sensitive to fixation.

\subsection{IEM Staining}

The strongest gold labeling was observed in materials fixed in $3 \% \mathrm{PF}+0.01 \% \mathrm{GA}+0.1 \% \mathrm{CaCl} 2$ in $0.05 \mathrm{M}$ Hepes-KOH buffer (pH 7.4), followed by the tissue fixed in 4\% PF alone. Gold signals showing TRA98 antigenic sites were localized to the nuclei of spermatogenic cells, including spermatogonia, spermatocytes, and round and elongated spermatids. The cytoplasm and the other organelles, including mitochondria, lysosomes, Golgi complexes, endoplasmic reticulum, and acrosome were very weakly or not labeled. Gold labeling was intensified by the silver enhancement method as if we view a whole cell, the gold particles were too small to identify. In the nuclei, silver grains seem to associate with some fibrous structures but not with the nucleolus (Figure 5(A)) When the silver grains closely neighboring each other were bound by lines, a few or no silver grains were present among the lines (Figure 5(B)). In the mid to late spermatocytes, the XY body was labeled more strongly than the other nuclear area (Figure 6(A), arrows). The granular component of the nucleolus closely associated with the XY body was negative for TRA98 (Figure 6(B), open arrow). Few or no signals were observed in the nu- 


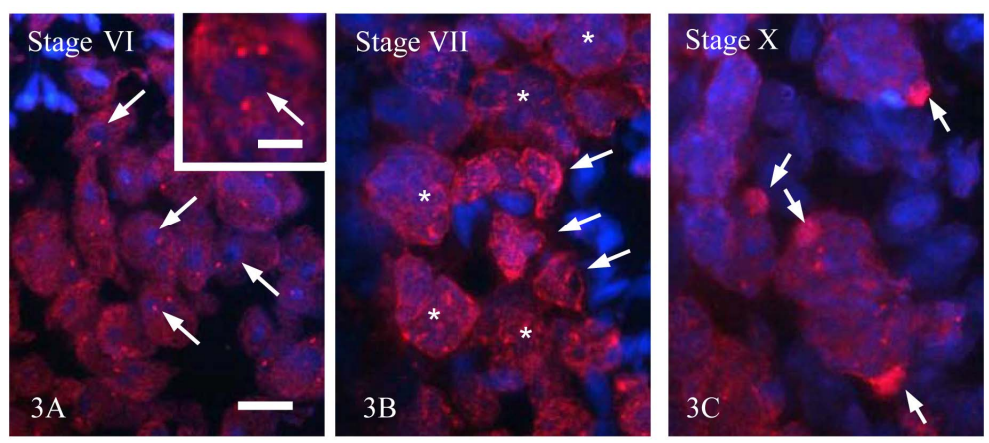

Figure 3. High power view of mouse testis sections stained for TRA98. (A) The nuclei of round spermatids are stained for TRA98.A reticular staining pattern is observed. In addition, strongly stained spots are located close to the TRA98-negative nuclear area (arrows). In the inset, they are shown at a higher magnification. (B) A reticular staining pattern is also observed in pachytene spermatocytes $(*)$ and in round spermatids (arrows); (C) The nuclei of pachytene spermatocytes are flecked with TRA98 staining and XY body-like structures are heavily stained (arrows). Magnification A-C: $\times 200$ and inset: $\times 450$. Bars for A-C, $25 \mu \mathrm{m}$; and for inset, $10 \mu \mathrm{m}$.
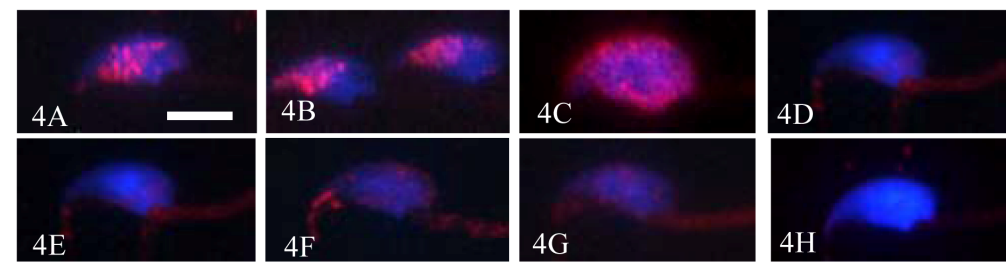

Figure 4. Sperm heads stained for TRA98 after various treatments. (A)-(D) Unfixed sperm. (E)-(H) Paraformaldehyde-fixed sperm. (A), (E) 4M urea treatment. (B), (F). 2 M guanidine hydrochloride. (C), (G) $2 \%$ mercaptoethanol. (D), (H). No treatment. Note that fixed materials and untreated ones are not stained for TRA98. Magnification $(\mathrm{A})-(\mathrm{H}): \times 1200 . \mathrm{Bar}=5 \mu \mathrm{m}$ for all figures.

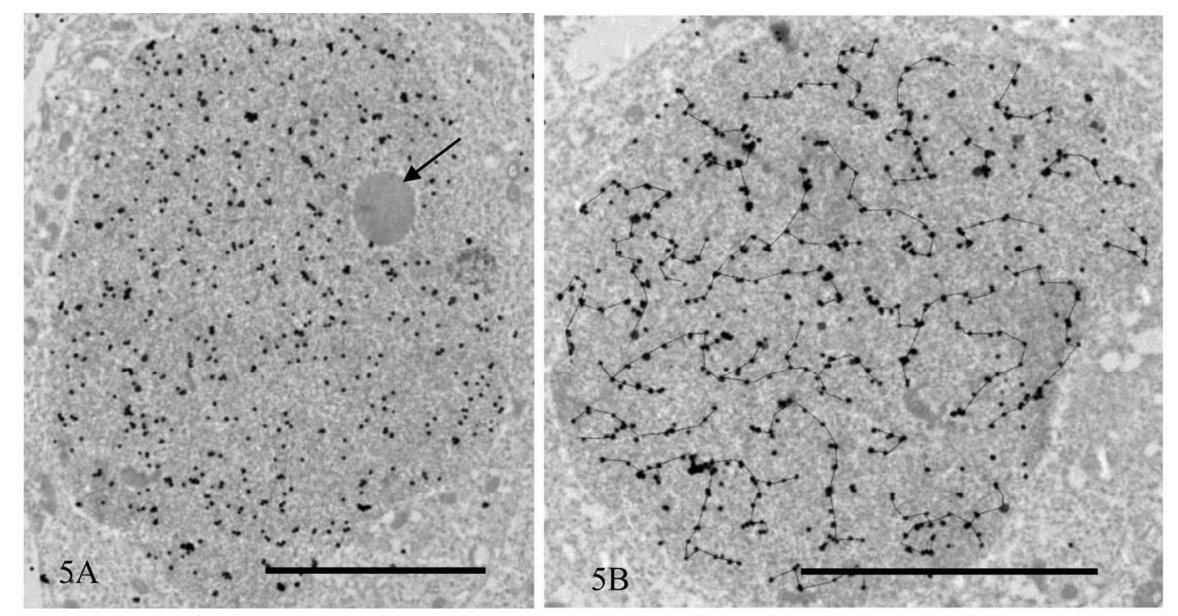

Figure 5. Immunogold staining of TRA98 after silver enhancement. (A) Spermatid at step 4. The acrosome is out of section. The nucleoplasm is heavily labeled but the nucleolus is not (arrow); (B) Spermatocyte at stage XI. Silver grains closely located are traced by lines. Note that the localization pattern is very similar to the reticular staining pattern observed by IF. Magnification (A): $\times 50,400$, and (B): $\times 74,000$. Bar $=5 \mu \mathrm{m}$.

clei of the Sertoli cells (Figure 6(B)).

We observed the spermatogenic cells at higher magnification in order to investigate the association of TRA98 with sub-nuclear structures using immunogold staining without silver enhancement. Gold particles showing TRA-
98 sites were closely associated with structures comprising dense fibrous materials and frequently gold particles were concentrated in some of these areas (Figure 7(A), circles). Furthermore, dense fibrous structures along synaptonemal complexes appearing in early to middle sper- 

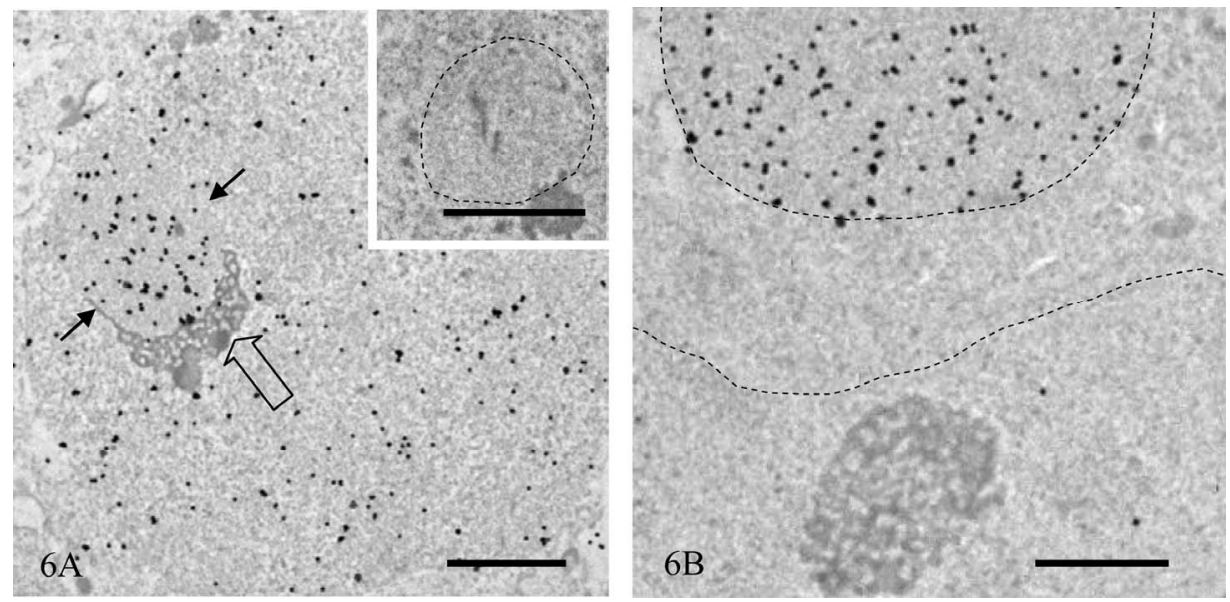

Figure 6. Immunogold staining for TRA98 after silver enhancement. (A) The nucleus of pachytene spermatocyte at stage VI. Many silver grains are scattered in the nucleoplasm and are much more concentrated in XY body (arrows). The granular component of the nucleolus closely associated with the XY body is negative for TRA98 (open arrow). Inset. Immunocytochemical control. Nucleoplasm including the XY body (enclosed by a dashed line) is shown. No silver grains are seen; (B) The nuclei (enclosed by a dashed line) of an early pachytene spermatocyte (SC) and of a Sertoli cell (Ser) are shown. Note that the former is strongly labeled whereas the latter is almost negative. Magnification $(\mathrm{A}): \times 9,000$, Inset: $\times 17,000$, and $(\mathrm{B}): \times 16,000$. Bar $=1 \mu \mathrm{m}$ for all.
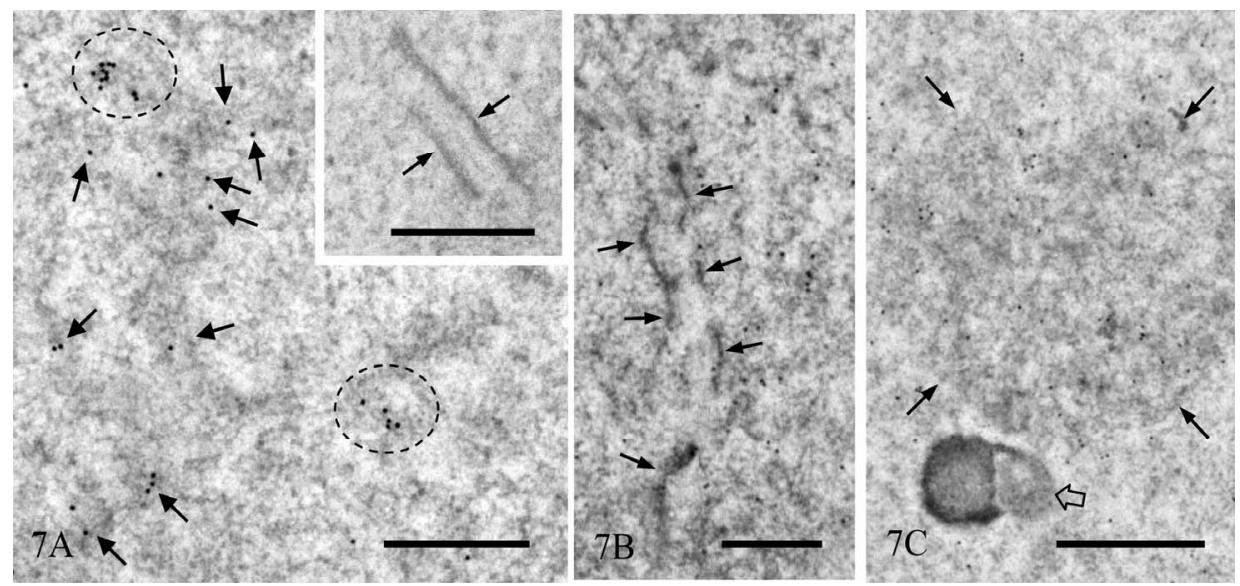

Figure 7. Immunogold staining for TRA98 without silver enhancement. (A) Nucleoplasm of spermatid at step 7. In some areas gold particles are concentrated (circles) and associated with dense amorphous structures (arrows). Inset. Immunocytochemical control. Nucleoplasm of mid pachy- tene spermatocyte, including synaptonemal complex (arrows) is shown. No gold particles are noted; (B) Nucleoplasm containing synaptonemal complex (arrows) is shown. Gold particles are associated with electron dense masses; (C) Nucleoplasm of a step 6 spermatid. The round aggregate of fibrous material is labeled more strongly than the other area. The nucleolus is not labeled (open arrow). Magnification (A): $\times 17,000$, Inset: $x$ 34,000, (B): $\times 24,000$, and $(C): \times 17,000$. Bar $=1 \mu \mathrm{m}$ for $(A)$ and $(C)$, and $=0.5 \mu \mathrm{m}$ for inset and (B).

matocytes were labeled (Figure 7(B)). In round spermatids at steps $4-7$, relatively large structures consisting of fine fibrous materials were labeled more strongly than the other nuclear area; small nucleoli of these cells were consistently negative for TRA98 (Figure 7(C)) These gold labels were not seen in the IEM control sections incubated with unrelated rat $\operatorname{IgG}$ fraction, followed by immunogold probe (Figure 7(A), inset). Labeling in the nuclei of late spermatids decreased, but a low level of labeling was maintained even in spermatozoa (Figure 8).

\subsection{Expression of TRA98 during Spermatogenesis Revealed by Quantitative IEM}

First we examined the changes in TRA98 expression in the developing spermatocytes. The labeling density increased gradually and reached the first peak at stage II-III. It decreased until stage VII. and then slightly increased again. The second peak of the labeling density was observed at stage XI (Figure 9). Next we observed 


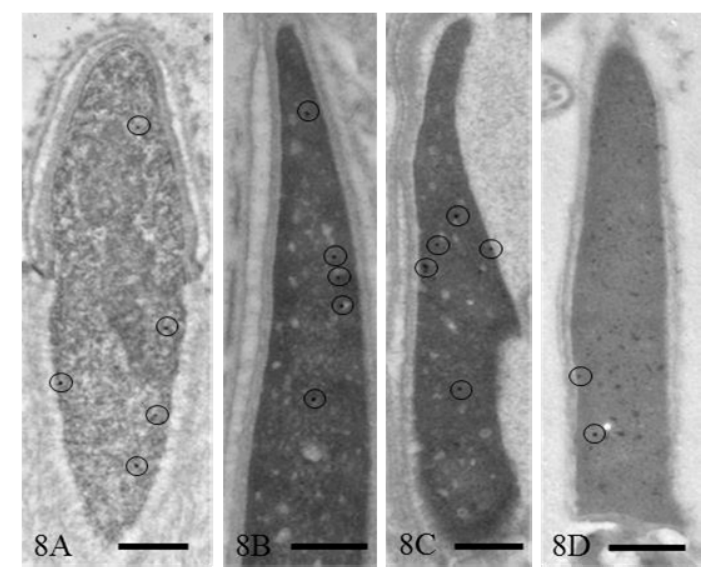

Figure 8. Immunogold staining for TRA98. A. Spermatid at step 10. B. Spermatid at step 13. C. Spermatid at step 16. D. Epididymal sperm. Gold particles showing TRA98 are enclosed by circles. Magnification A: $\times 18,000, B: \times 20,000, C$ : $\times 18,000$, and $D: \times 20,000$. Bar $=0.5 \mu \mathrm{m}$.

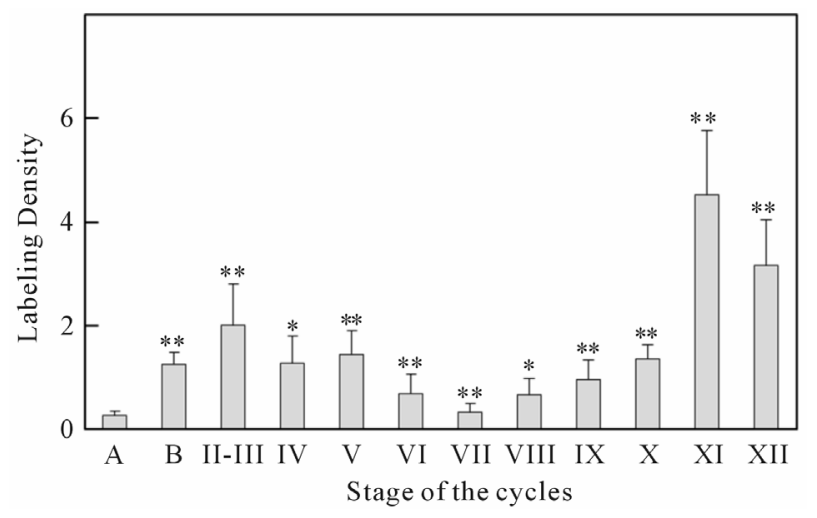

Figure 9. Expression of TRA98 in spermatogonia A and B, and developing spermatocytes revealed by quantitative IEM. Results are the mean \pm SEM of 10 micrographs. ${ }^{*} P<$ $0.05, * * P<0.01$ as compared with spermatogonia A with the Student's $t$-test.

the change in labeling density during differentiation of spermatids. As shown in Figure 10, the labeling density increased gradually and reached the maximum at step 5 . The labeling density then decreased slowly, but did not reach zero in epididymal sperm.

\section{Discussion}

\subsection{Western Blotting}

TRA98 monoclonal antibody developed a band with molecular mass of $100 \mathrm{kDa}$ in the nuclear extract. Western and dot blotting of cell fractions from mouse testis show it to be distributed almost exclusively in the nuclear fraction. A trace small amount of TRA98 was detected in the microsomes. This might be due to nascent

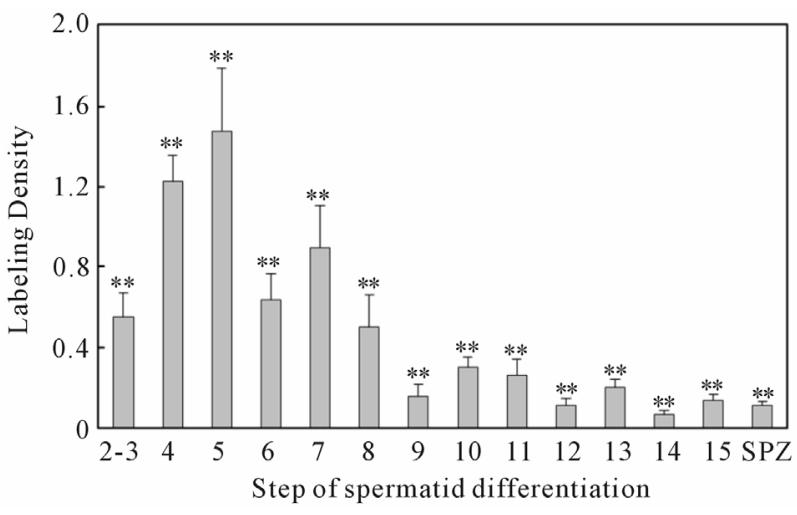

Figure 10. Expression of TRA98 in developing spermatids revealed by quantitative IEM. Data are taken from spermatids at steps $2-3$ to 16 and epididymal sperm (SPZ). Results are the mean \pm SEM of 10 micrographs. ${ }^{*} \boldsymbol{P}<0.05$, $* * P<0.01$ as compared with spermatogonia with the Student's t-test.

TRA98 still binding to free ribosomes centrifuged into microsomes. Although we have not yet obtained a clear signal in the nuclear extract of epididymal sperm by Western blotting, our immunostaining showed a distinct signal in sperm head. This means that TRA98 protein is carried to the egg after fertilization, where it seems to have some function. TRA98 in sperm was not immunostained after fixation, followed by routine detergent treatment but unfixed sperm were stained after treatment with a high concentration of urea and guanidine hydrochloride or reduction with mercaptoethanol. From IEM, staining signals decreased as elongated spermatids differentiated. These results suggest that TRA98 strongly binds with nuclear materials and is masked, such that antibody is not able to access to it.

\subsection{IF Staining of TRA98}

IF staining showed that the nuclei of all spermatogenic cells were positively stained for TRA98 with the exception of late elongated spermatids. The results strongly suggest that TRA98 is a testis-specific nucleus-resident protein, as described by Tanaka [5]. The present study showed that TRA98 was not homogeneously localized in the nucleus but showed a reticular staining pattern. This means that TRA98 is associated with some sub-nuclear structures. It is likely that the structures are euchromatin scattered in the nucleoplasm. In addition to the reticular staining pattern, discrete spots and XY body-like structures were strongly stained in the nuclei of round spermatids and spermatocytes, respectively. These structures were not present in elongated spermatids. The results suggest that TRA98 functions in these structures during particular stages or steps. From quantitative analysis of TRA98 expression during spermatogenesis, the function 
of TRA98 seems to be elevated first at stage II - III and next at stage XI.

The negative staining error was dissolved by improvement of immunostaining. When smear preparations of unfixed epididymal sperm were treated with denaturing reagents such as urea and guanidine hydrochloride or with mercaptoethanol, sperm heads were stained for TRA98. These pretreatments were not efficient after fixation with PF. After treatment with mercaptoethanol, sperm heads were swollen to approximately twofold the original size and stained strongly. The present results of IF staining indicate that TRA98 is contained in the mature sperm head.

\subsection{IEM Staining}

IEM staining confirmed the results of IF. Signals for TRA98 were detected in the nuclei of all spermatogenic cells with varied staining intensities. The nuclei of elongated spermatids and epididymal sperm were weakly stained, although they were almost negative by IF staining. This is due to the fact that the IEM reaction is performed on the surface of thin sections where some epitopes are always exposed. Gold signals were associated with dense materials located in the nucleoplasm, and consequently exhibited a reticular staining pattern, very similar to that of bromodeoxyuridine-incorporated DNA [11]. The results suggest that TRA98 is bound with subnuclear structures. From the similarities of the distribution patterns between TRA98 and bromodeoxyuridine, TRA98 seems to be associated with DNA or DNA-related proteins. The other remarkable nuclear site of TRA98 is the XY body-like structure, which was labeled more strongly than the other parts. The XY body appears in mid and late spermatocytes and its fine structure has been described in detail by Solari [12] and Knibiehler et al. [13]. According to these authors, it is located at the periphery of the nucleus and is sometimes accompanied by electron-dense heterochromatin. The TRA98-localized structures have these morphological characteristics. Many proteins have been reported to be associated with the XY body, including Xlr-related and meiosis regulated protein (Xmr) [14,15], Mr 51,000 [16], XY bodyassociated protein 40 (XY40) [17], XY body-associated protein 77 (XY77) [18], NIMA (never in mitosis gene a)-related kinase 2 (Nek2) [19], germ cell nuclear factor (GCNF) [20], murine heterochromatin protein (M31) [21], macroH2A [22], XY body protein (XYbp) [23], Histone-lysine N-methyltransferase (Suv 39h2) [24], asynaptin (ASY) [25], SUMO modified proteins [26], Doublesex/MAB-3 product (DMRT7) [27], and synaptonemal complex protein 1 (Sycp1) [28]. Despite the discovery of these proteins, the function of the $\mathrm{XY}$ body is still unclear. Similarly, the function of TRA98 in the XY body is unknown. Our study suggests that the XY body is one of the functional sites of TRA98 in the nucleus.

\subsection{Expression of TRA98 during Spermatogenesis}

During spermatocyte development, TRA98 was expressed in all stages and two peaks were noted. The first peak appeared at stage II-III and the second peak occurred at stage XI, which was approximately twice as higher as the first peak. The result suggests that the functional activity of TRA98 increases at these stages. From the early to the late differentiation period of pachytene spermatocytes, testis-specific histones, tH2A and tH2B, are lost [29], and, instead, H1t appears [30]. Thus, nuclear proteins of the testis are dynamically exchanged. TRA98 exists continuously within the nucleus of differentiating spermatocytes though in varying amounts. This leads us to consider that TRA98 may be involved in more general functions such as the regulation of transcription or silencing of genes. In relation to this, it is interesting that TRA98 was expressed most strongly in diplotene spermatocytes because in successive meiosis, germ cells should maintain genetic and chromosomal stability from genetic intruders, retrotransposons, so that TRA98 is likely to play some role in silencing of retrotransposons.

During spermiogenesis, TRA98 increases until step 5 and then gradually decreases. It has been reported that many nuclear proteins appears and disappears during spermiogenesis [31]. For example, spermatid-specific histone $2 \mathrm{~b}, \mathrm{ssH} 2 \mathrm{~B}$, is expressed in late spermatocytes and disappears in spermatids at steps $5-8$ [32]. Histone H1-like protein, Hanp1/H1T2, exists from step 5 to step 13 [33]. Spermatid-specific linker histone H1-like protein, Hils1, appears at step 9 and disappears at step 13 [34]. TNP1 and TNP2 appear at around step 12 and are replaced by protamine 1 and 2 [35]. The expression peak of TRA98 in spermatids overlaps with the end of ssH2B expression and the beginning of Hanp1 expression. Thus, there is no complete overlap of TRA98 expression with the other nuclear proteins, so the function of TRA98 seems not to be related to the functions of the other nuclear proteins reported.

\section{Acknowledgements}

The work was supported by the University research fund, in part by a grant-in-aid (17570158) from the Ministry of Education, Science, Culture and Sport, and by the Science Research Promotion Fund from the Promotion and Mutual Aid Corporation for Private Schools of Japan. 


\section{References}

[1] E. M. Eddy, "Germ Plasm and the Differentiation of Germ Cell Line," International Review of Cytology, Vol. 43, 1975, pp. 229-280. doi:10.1016/S0074-7696(08)60070-4

[2] D. M. de Krester and J. B. Kerr, "The Cytology of the Testis," In: E. Knobil and J. Neill, Eds., The Physiology of Reproduction, Raven Press, New York, 1988, pp. 837932.

[3] Y. Clermont, R. Oko and L. Hermo, "Cell Biology of Mammalian Spermatogenesis," In: C. Desjardins and L. L. Ewing, Eds., Cell and Molecular Biology of the Testis, Oxford University Press, New York, 1993, pp. 332-376.

[4] N. B. Hecht, "Gene Expression During Male Germ Cell Development," In: C. Desjardins and L. L. Ewing, Eds., Cell and Molecular Biology of the Testis, Oxford University Press, New York, 1993, pp. 400-432.

[5] H. Tanaka, L. A. V. D. Pereira, M. Nozaki, J. Tsuchida, K. Sawada, H. Mori And Y. Nishimune, "A Germ CellSpecific Nuclear Antigen Recognized by a Monoclonal Antibody Raised against Mouse Testicular Germ Cells," International Journal of Andrology, Vol. 20, No. 6, 1997, pp. 361-366. doi:10.1046/j.1365-2605.1998.00080.x

[6] C. de Roe, P. J. Courtoy and P. Baudhuin, "A Model of Protein A-Colloidal Gold Interactions," Journal of Histochemistry and Cytochemistry, Vol. 35, No. 11, 1987, pp. 1191-1198. doi:10.1177/35.11.3655323

[7] C. M. Haraguchi, T. Mabuchi and S. Yokota, "Localization of a Mitochondrial Type of NADP-Dependent Isocitrate Dehydrogenase in Kidney and Heart of Rat: An Immunocytochemical and Biochemical Study," Journal of Histochemistry and Cytochemistry, Vol. 51, No. 2, 2003, pp. 215-226. doi:10.1177/002215540305100210

[8] D. Rickwood and T. C. Ford, "Preparation and Fractionation of Nuclei, Nucleoli and Deoxyribonucleoproteins," In: D. Rickwood, Ed., Iodinated Density Gradient Media. A Practical Approach, IRL Press, Oxford, 1983, pp. 69-89.

[9] L. D. Russell, R. A. Ettlin, A. P. S. Hikim and E. D. Clegg, "Histological and Histopathological Evaluation of the Testis," Cache River Press, Florida, 1990, pp. 120-161.

[10] E. R. Weibel, G. S. Kistler and W. F. Scherle, "Practical Stereological Methods for Morphometric Cytology," Journal of Cell Biology, Vol. 30, No. 1, 1966, pp. 23-38. doi: $10.1083 /$ jcb.30.1.23

[11] R. Tamatani, Y. Taniguchi and Y. Kawarai, "Ultrastructural Study of Proliferating Cells with an Improved Immunocytochemical Detection of DAN-Incorporated Bromodeoxyuridine," Journal of Histochemistry and Cytochemistry, Vol. 43, 1995, pp. 21-29. doi: $10.1177 / 43.1 .7822760$

[12] A. J. Solari, "The Evolution of the Ultrastructure of the Sex Chromosomes (Sex vesicle) during Meiotic Prophase in Mouse Spermatocytes," Journal of Ultrastructure Research, Vol. 27, No. 3-4, 1969, pp. 289-305. doi:10.1016/S0022-5320(69)80018-3

[13] B. Knibiehler, C. Mirre, M. Hartung, P. Jean and A. Stahl, "Sex Vesicle-Associated Nucleolar Organizers in Mouse
Spermatocytes: Localization, Structure, and Function," Cytogenetics and Cell Genetics, Vol. 31, No. 2, 1981, pp. 47-57.

[14] A. Calenda, B. Allenet, D. Escalier, J.-F. Bach and H.-J., Garchon, "The Meiosis-Specific Xmr Gene Product is Homologous to the Lymphocyte Xlr Protein and is a Component of the XY Body," EMBO Journal, Vol. 13, 1994, pp. 100-109.

[15] D. Escalier and H.-J. Garchon, "XMR is Associated with the Asynapsed Segments of Sex Chromosomes in the XY Body of Mouse Primary Spermatocytes," Chromosoma, Vol. 109, No. 4, 2000, pp. 259-265. doi: $10.1007 / \mathrm{s} 004120000075$

[16] A. Smith and R. Benavente, "An Mr 51,000 Protein of Mammalian Spermatogenic Cells that is Common to the Whole XY Body and Centromeric Heterochromatin of Autosomes," Chromosoma, Vol. 103, No. 9, 1995, pp. 591-596. doi:10.1007/BF00357685

[17] M. Alsheimer, Y. Imamichi, H. Heid and R. Benavente, "Molecular Characterization and Expression Pattern of XY Body-Associated Protein XY40 of the Rat," Chromosoma, Vol. 106, No. 5, 1997, pp. 308-314. doi:10.1007/s004120050252

[18] M. Kralewski, A. Nevello and R. Benavente, "A Novel Mr 77,000 Protein of the XY Body of Mammalian Spermatocytes: Its Localization in Normal Animals and in Searle's Translocation Carriers," Chromosoma, Vol. 106, No. 3, 1997, pp. 160-167. doi:10.1007/s004120050235

[19] K. Rhee and D. J. Wolgemuth, "The NIMA-Related Kinase 2, Nek2, is Expressed in Specific Stages of the Meiotic Cell Cycle and Associates with Meiotic Chromosomes," Development, Vol. 124, No. 11, 1997, pp. 21672177.

[20] U.-M. Bauer, S. Schneider-Hirsch, S. Reinhardt, R. Benavente and A. Maelicke, "The Murine Nuclear Orphan Receptor GCNF is Expressed in the XY Body of Primary Spermatocytes," FEBS Letters, Vol. 439, No. 3, 1998, pp. 208-214. doi:10.1016/S0014-5793(98)01327-1

[21] D. Motzkus, P. B. Singh and S. Hoyer-Fender, "M31, a Murine Homolog of Drosophila HP1, is Concentrated in the XY Body during Spermatogenesis," Cytogenetics and Cell Genetics, Vol. 86, No. 1, 1999, pp. 83-88. doi: $10.1159 / 000015418$

[22] S. Hoyer-Fender, C. Costanzi and J. R. Pehrson, "Histone macroH2A1.2 is Concentrated in the XY Body by the Early Pachytene Stage of Spermatogenesis," Experimental Cell Research, Vol. 258, No. 2, 2000, pp. 254-260. doi:10.1006/excr.2000.4951

[23] M. Párraga and J. del Mazo, "XYbp, a Novel RINGFinger Protein, is a Component of the XY Body of Spermatocytes and Centrosomes," Mechanisms of Development, Vol. 90, No. 1, 2000, pp. 95-101. doi:10.1016/S0925-4773(99)00223-3

[24] D. O'Carroll, H. Scherthan, A. H. F. M. Peters, S. Opravil, A. R. Haynes, G. Laible, S. Rea, M. Schmid, A. Lebersorger, M. Jerratsch, L. Sattler, M. G. Mattel, P. Denny, S. D. M. Brown, D. Schweizer and T. Jenuwein, "Isolation and Characterization of Suv39h2, a Second 
Histone H3 Methyltransferase Gene that Displays Testis-Specific Expression," Molecular and Cellular Biology, Vol. 20, No. 24, 2000, pp. 9423-9433. doi:10.1128/MCB.20.24.9423-9433.2000

[25] J. M. A. Turner, S. K. Mahadevaiah, R. Benevente, H. H. Offenberg, C. Heyting and P. S. Burgoyne, "Analysis of Male Meiotic 'Sex Body' Proteins During XY Female Meiosis Provides New Insights into Their Functions," Chromosoma, Vol. 109, 2000, pp. 426-432. doi:10.1007/s004120000097

[26] R. S. Rogers, A. Inselman, M. A. Handel and M. J. Matunis, "SUMO Modified Proteins Localize to the XY Body of Pachytene Spermatocytes," Chromosoma, Vol. 113, No. 5, 2004, pp. 233-243. doi:10.1007/s00412-004-0311-7

[27] S. Kim, S. H. Namekawa, L. M. Niswander, J. O. Ward, J. T. Lee, V. J. Bardwell and D. Zarkower, "A Mammal-Specific Doublesex Homolog Assocaites with Male Sex Chromatin and Is Required for Male Meiosis," PLos Genetics, Vol. 3, No. 4, 2007, pp. 559-571. doi:10.1371/journal.pgen.0030062

[28] F. A. T. de Vries, E. de Boer, M. van den Bosch, W. M. Baarends, M. Ooms, L. Yuan, J.- G. Liu, A. A. van Zeeland, C. Heyting and A. Pastink, "Mouse Sycp1 Functions in Synaptonemal Complex Assembly, Meiotic Recombination, and XY Body Formation," Gene Development, Vol. 18, No. 1, 2011, pp. 1376-1389.

[29] M. L. Meistrich, L. R. Bucci, P. K. Trosite-Weige and W. A. Brock, "Histone Variants in Rat Spermatogonia and Primary Spermatocytes," Developmental Biology, Vol.
112, No. 1, 1985, pp. 230-240. doi:10.1016/0012-1606(85)90137-X

[30] B. Drabent, C. Bode and D. Doenecke, "Structure and Expression of the Mouse Testicular H1 Histone Gene (H1t)," Biochimica et Biophysica Acta, Vol. 1216, No. 2, 1993, pp. 311-313.

[31] E. M. Eddy, "Regulation of Gene Expression During Spermatogenesis," Seminars in Cell and Developmental Biology, Vol. 9, No. 4, 1998, pp. 451-457. doi:10.1006/scdb.1998.0201

[32] S. B. Moss and J. M. Orth, "Localization of a SpermatidSpecific Histone 2B Protein in Mouse SpermatoGenic Cells," Biology of Reproduction, Vol. 48, No. 5, 1993, pp. 1047-1056. doi:10.1095/biolreprod48.5.1047

[33] H. Tanaka, N. Iguchi, A. Isotani, K. Kitamura, Y. Toyama, Y. Matsuoka, M. Onishi, K. Masai, M. Maekawa, K. Toshimori, M. Okabe and Y. Nishimune, "HANP1/ H1T2, a Novel Histone H1-Like Protein Involved in Nuclear Formation and Sperm Fertility," Molecular Cell Biology, Vol. 25, No. 16, 2005, pp. 7107-7119. doi:10.1128/MCB.25.16.7107-7119.2005

[34] Y.-N. Teng, P.-L. Kuo, T.-C. Cheng and M.-H. Liao, "Histone Gene Expression Profile During Spermatogenesis," Fertility and Sterility, Vol. 93, No. 7, 2010, pp. 2447 2449. doi:10.1016/j.fertnstert.2009.09.016

[35] K. Steger, K. Pauls, T. Klonisch, F. E. Franke and M. Bergmann, "Expression of Protamine-1 and -2 mRNA During Human Spermatogenesis," Molecular Human Reproduction, Vol. 6, 2000, pp. 219-225. doi:10.1093/molehr/6.3.219 